Bull. Korean Math. Soc. 50 (2013), No. 6, pp. 1827-1840

http://dx.doi.org/10.4134/BKMS.2013.50.6.1827

\title{
QUALITATIVE ANALYSIS OF A DIFFUSIVE FOOD WEB CONSISTING OF A PREY AND TWO PREDATORS
}

\author{
HONG-Bo SHI
}

\begin{abstract}
This paper is concerned with the positive steady states of a diffusive Holling type II predator-prey system, in which two predators and one prey are involved. Under homogeneous Neumann boundary conditions, the local and global asymptotic stability of the spatially homogeneous positive steady state are discussed. Moreover, the large diffusion of predator is considered by proving the nonexistence of non-constant positive steady states, which gives some descriptions of the effect of diffusion on the pattern formation.
\end{abstract}

\section{Introduction}

Due to the universal existence of energy transformation, predator-prey system is very important in describing the population evolution, and it is one of the dominant themes in both ecology and mathematical ecology. Because of the differences in capturing food and consuming energy, a major trend in theoretical work on predator-prey dynamics has been launched so as to derive more realistic models and functional responses, for example, Lotka-Volterra type $[22,31]$, Holling type [14], Beddington-DeAngelis type $[3,5]$ and so on. These models often involve only one predator species and one prey species. In reality, it is very common that one species is captured by several species in food chains. In particular, Hixon and Jones [13] found that the density-dependent mortality in demersal marine fishes is often caused by interaction of predation and competition. In a very recent paper, to study how the nonlinear mortality rate determines the dynamics of such competition models, Ruan et al. [29] proposed the following two predators and one prey model with Holling II type

Received February 5, 2012.

2010 Mathematics Subject Classification. 35J60, 37B25, 92D25.

Key words and phrases. predator-prey system, positive steady state, large diffusion, Holling II type functional response, local/global asymptotic stability.

Supported by Universities Natural Science Foundation of Jiangsu Province of China (No. 11KJB110003) and Young Talents Support Projects of Huaiyin Normal University (11HSQNZ02). 
functional response:

$$
\left\{\begin{array}{l}
\frac{\mathrm{d} u}{\mathrm{~d} t}=r u\left(1-\frac{u}{K}\right)-\frac{a u v}{1+b u}-\frac{A u w}{1+B u} \\
\frac{\mathrm{d} v}{\mathrm{~d} t}=v\left(-d+\frac{e u}{1+b u}\right) \\
\frac{\mathrm{d} w}{\mathrm{~d} t}=w\left(-D-G w+\frac{E u}{1+B u}\right)
\end{array}\right.
$$

where $u$ represents the density of the prey, $v, w$ reflect the densities of two predators, respectively. In the third equation, $G w^{2}$ denotes the density dependent mortality term for the second species and is also referred to as a 'closure term', which describes either a self-limitation of $w$ or the influence of predation. In ecology, self-limitation can occur if another factor besides food can possibly become limiting at high population densities. Predation on consumers can increase as the $w^{2}$ power if higher consumer densities attract greater attention from predators or if consumers become more vulnerable at higher densities. Obviously, all the constants, i.e., $r, a, b, d, e, A, B, E, D, G, K$, are positive for their biological sense. Note that both $v$ and $w$ capture the food $u$, of which the density is limit, $v$ and $w$ are essentially competitive each other. In such a sense, (1.1) formulates the dynamics of two competitive predators and one prey. In [29], the authors mainly investigated the stability of positive constant equilibrium of (1.1) and gave some numerical simulations.

Applying the following scaling to system (1.1),

$$
\begin{aligned}
& \frac{u}{K} \mapsto u, v \mapsto v, w \mapsto w, r t \mapsto t, \frac{a}{r} \mapsto a, b K \mapsto b, \frac{A}{r} \mapsto A, B K \mapsto B, \\
& \frac{d}{r} \mapsto d, \frac{e K}{d} \mapsto e, \frac{D}{r} \mapsto D, \frac{G}{D} \mapsto G, \frac{E K}{D} \mapsto E,
\end{aligned}
$$

then it can be simplified as follows,

$$
\left\{\begin{array}{l}
\frac{\mathrm{d} u}{\mathrm{~d} t}=u(1-u)-\frac{a u v}{1+b u}-\frac{A u w}{1+B u} \\
\frac{\mathrm{d} v}{\mathrm{~d} t}=d v\left(-1+\frac{e u}{1+b u}\right) \\
\frac{\mathrm{d} w}{\mathrm{~d} t}=D w\left(-1-G w+\frac{E u}{1+B u}\right)
\end{array}\right.
$$

For the sake of convenience, in the remainder of this paper, we also denote $\mathbf{u}=(u, v, w)^{T}$ and

$$
\mathbf{G}(\mathbf{u})=\left(\begin{array}{l}
G_{1}(\mathbf{u}) \\
G_{2}(\mathbf{u}) \\
G_{3}(\mathbf{u})
\end{array}\right)=\left(\begin{array}{c}
u g_{1}(\mathbf{u})=u\left(1-u-\frac{a v}{1+b u}-\frac{A w}{1+B u}\right) \\
v g_{2}(\mathbf{u})=d v\left(-1+\frac{e u}{1+b u}\right) \\
w g_{3}(\mathbf{u})=D w\left(-1-G w+\frac{E u}{1+B u}\right)
\end{array}\right) .
$$

It is evident that (1.2) has a positive equilibrium if

$$
\left\{\begin{array}{l}
e>b, \quad E-B>e-b, \\
G e(e-b-1)(B+e-b)^{2}-A e(e-b)^{2}[(E-B)-(e-b)]>0 .
\end{array}\right.
$$


The positive equilibrium is uniquely given by

$$
\begin{aligned}
& u^{*}=\frac{1}{e-b}, \quad v^{*}=\frac{G e(e-b-1)(B+e-b)^{2}-A e(e-b)^{2}[(E-B)-(e-b)]}{G a(e-b)^{2}(B+e-b)^{2}}, \\
& w^{*}=\frac{(E-B)-(e-b)}{G(B+e-b)} .
\end{aligned}
$$

In what follows, we always denote the unique positive equilibrium by $\mathbf{u}^{*}=$ $\left(u^{*}, v^{*}, w^{*}\right)^{T}$.

However, in some cases, the spatial distribution is very important in population dynamics, such as the species invasion [1] and spreading of epidemic [6]. One typical and classical method is to describe the spatial migration of individuals by Laplace operator (see $[4,9]$ ) and such an operator can give rise to some important spatiotemporal pattern, for example Turing instability (or the diffusion-driven instability, see [30]). Particularly, during the past decades, diffusive predator-prey systems have been extensively studied, and one can refer to $[10,15,19,20,24,25,26,27,28,32]$ and references cited therein.

If we take into account the inhomogeneous distribution of the predators and prey in different spatial locations at any given time and assume that the predator-prey system is self-contained with zero population flux across the boundary in (1.2), then we are led to consider the following PDE system of reaction-diffusion type:

$$
\begin{cases}\frac{\partial u}{\partial t}-d_{1} \Delta u=G_{1}(\mathbf{u}), & x \in \Omega, t>0, \\ \frac{\partial v}{\partial t}-d_{2} \Delta v=G_{2}(\mathbf{u}), & x \in \Omega, t>0, \\ \frac{\partial w}{\partial t}-d_{3} \Delta w=G_{3}(\mathbf{u}), & x \in \Omega, t>0, \\ \frac{\partial u}{\partial \nu}=\frac{\partial v}{\partial \nu}=\frac{\partial w}{\partial \nu}=0, & x \in \partial \Omega, t>0, \\ u(x, 0)=u_{0}(x) \geq 0, v(x, 0)=v_{0}(x) \geq 0, & \\ w(x, 0)=w_{0}(x) \geq 0, & x \in \bar{\Omega},\end{cases}
$$

where $\Omega \subset \mathbb{R}^{N}$ is a bounded domain with smooth boundary $\partial \Omega, \nu$ is the outward unit normal vector of the boundary $\partial \Omega$. The constants $d_{1}, d_{2}$, and $d_{3}$, called diffusion coefficients, are positive, and the initial data $u_{0}(x), v_{0}(x)$, and $w_{0}(x)$ are continuous functions. It is obvious that $\mathbf{u}^{*}$ is the unique positive constant steady state of (1.4).

In the case that $w \equiv 0$ (or the species formulated by the third equation vanishes in population dynamics), system (1.4) reduces to a two species predatorprey model with Holling II type functional response. Ko and Ryu [16] studied a Holling II type predator-prey model incorporating a prey refuge under homogeneous Neumann boundary condition, in which the large time behavior of time-dependent system, existence and nonexistence of nonconstant positive steady states are concerned. Furthermore, Ko and Ryu [17, 18] investigated a system (1.4) with $w=0$ and general functional response $p(u)$ (monotonic or nonmonotonic) and established the existence and nonexistence of nonconstant positive steady states. Du and Lou $[7,8]$ considered a system similar to (1.4) 
with $w=0$ under Dirichlet and Neumann boundary condition, respectively. They discussed the existence and nonexistence of nonconstant positive steady states.

If $v=0, a=E=1$ in system (1.1), then we can get a predator-prey model proposed by Bazykin [2], which was also considered in [33]. More precisely, the authors of [33] introduced diffusion and ratio-dependence into this model under homogeneous Neumann boundary condition, and gave the global asymptotic stability of positive constant steady state by using iteration method.

Motivated by the works mentioned above, the main purpose of this article is concerned with positive steady states of system (1.4). We first consider the constant steady state by proving its asymptotic stability. As we all know, in ecological models, different diffusions may play essentially different roles in determining the pattern formation (see [28]). Our another goal is to explore the effect of diffusion, namely, we shall consider the nonexistence of nonconstant positive solutions to the following elliptic system:

$$
\begin{cases}-d_{1} \Delta u=G_{1}(\mathbf{u}), & x \in \Omega, \\ -d_{2} \Delta v=G_{2}(\mathbf{u}), & x \in \Omega, \\ -d_{3} \Delta w=G_{3}(\mathbf{u}), & x \in \Omega, \\ \frac{\partial u}{\partial \nu}=\frac{\partial v}{\partial \nu}=\frac{\partial w}{\partial \nu}=0, & x \in \partial \Omega .\end{cases}
$$

Our results will show the importance of diffusion rate of the species in system (1.5). The large diffusion rate of the prey $u$ can lead to the nonexistence of spatially nonconstant positive solutions.

The rest of this paper is arranged as follows. In Section 2, we will investigate the local/global stability of positive constant steady state $\mathbf{u}^{*}$ with respect to time-dependent system (1.4). In Section 3, a priori estimates for positive solutions of elliptic system (1.5) and the nonexistence of nonconstant positive solutions to (1.5) is established. The last section is devoted to give some biological explanations for the obtained results.

\section{Asymptotic stability of $\mathbf{u}^{*}$}

In this section, we study the local/global asymptotic stability of the constant positive steady state $\mathbf{u}^{*}$ for reaction-diffusion system (1.4).

Before developing our argument, let us set up the following notations.

(i) $0=\mu_{0}<\mu_{1}<\mu_{2}<\cdots \rightarrow \infty$ are the eigenvalues of $-\Delta$ on $\Omega$ under homogeneous Neumann boundary condition.

(ii) $E\left(\mu_{i}\right)$ is the space of eigenfunctions corresponding to $\mu_{i}$ for $i=0,1,2, \ldots$.

(iii) $\mathbf{X}_{i j}:=\left\{\mathbf{c} \cdot \varphi_{i j}: \mathbf{c} \in \mathbb{R}^{2}\right\}$, where $\left\{\varphi_{i j}\right\}$ are an orthonormal basis of $E\left(\mu_{i}\right)$ for $i=0,1,2, \ldots$ and $j=1,2, \ldots, \operatorname{dim}\left[E\left(\mu_{i}\right)\right]$.

(iv) $\mathbf{X}:=\left\{\mathbf{u}=(u, v) \in\left[C^{1}(\bar{\Omega})\right]^{2}: \frac{\partial u}{\partial \nu}=\frac{\partial v}{\partial \nu}=0\right\}$, and so

$$
\mathbf{X}=\bigoplus_{i=1}^{\infty} \mathbf{X}_{i}, \quad \text { where } \quad \mathbf{X}_{i}=\bigoplus_{j=1}^{\operatorname{dim}\left[E\left(\mu_{i}\right)\right]} \mathbf{X}_{i j}
$$


To study the stability of $\mathbf{u}^{*}$ (the existence of $\mathbf{u}^{*}$ is clear by (1.3)), we consider the following linearized system of (1.4) at $\mathbf{u}^{*}$,

$$
\mathbf{u}_{t}=\left(\mathcal{D} \Delta+\mathbf{G}_{\mathbf{u}}\left(\mathbf{u}^{*}\right)\right) \mathbf{u},
$$

where $\mathbf{u}=(u(x, t), v(x, t), w(x, t))^{T}, \mathcal{D}=\operatorname{diag}\left\{d_{1}, d_{2}, d_{3}\right\}$, and

$$
\mathbf{G}_{\mathbf{u}}\left(\mathbf{u}^{*}\right)=\left(\begin{array}{ccc}
a_{11} & a_{12} & a_{13} \\
a_{21} & 0 & 0 \\
a_{31} & 0 & a_{33}
\end{array}\right)
$$

with

$$
\begin{aligned}
& a_{11}=\frac{G(B+e-b)^{3}[b(e-b-1)-e]+A(e-b)^{3}(B-b)[(E-B)-(e-b)]}{G e(e-b)(B+e-b)^{3}}, \\
& a_{12}=-\frac{a u^{*}}{1+b u^{*}}<0, \quad a_{13}=-\frac{A u^{*}}{1+B u^{*}}<0, \quad a_{21}=\frac{d e v^{*}}{\left(1+b u^{*}\right)^{2}}>0, \\
& a_{31}=\frac{D E w^{*}}{\left(1+B u^{*}\right)^{2}}>0, \quad a_{33}=-G D w^{*}<0 .
\end{aligned}
$$

From the definition of $a_{11}$, if

$$
G(B+e-b)^{3}[b(e-b-1)-e]+A(e-b)^{3}(B-b)[(E-B)-(e-b)]<0,
$$

then $a_{11}<0$ holds. For each $i=0,1,2, \ldots$, it is clear that $\mathcal{D} \Delta+\mathbf{G}_{\mathbf{u}}\left(\mathbf{u}^{*}\right)$ : $\mathbf{X}_{i} \rightarrow \mathbf{X}_{i}$. Moreover, $\lambda$ is an eigenvalue of this operator on $\mathbf{X}_{i}$ if and only if it is an eigenvalue of the matrix $M_{i}=-\mu_{i} \mathcal{D}+\mathbf{G}_{\mathbf{u}}\left(\mathbf{u}^{*}\right)$. The characteristic equation of $M_{i}$ is given by

$$
\psi_{i}(\lambda)=\lambda^{3}+B_{1 i} \lambda^{2}+B_{2 i} \lambda+B_{3 i}=0,
$$

in which

$$
\begin{aligned}
B_{1 i}= & \mu_{i}\left(d_{1}+d_{2}+d_{3}\right)-\left(a_{11}+a_{33}\right) \\
B_{2 i}= & \mu_{i}^{2}\left(d_{1} d_{2}+d_{2} d_{3}+d_{1} d_{3}\right)-\mu_{i}\left[a_{33} d_{1}+\left(a_{11}+a_{33}\right) d_{2}+a_{11} d_{3}\right] \\
& +a_{11} a_{33}-a_{12} a_{21}-a_{13} a_{31} \\
B_{3 i}= & \mu_{i}^{3} d_{1} d_{2} d_{3}-\mu_{i}^{2}\left(d_{1} d_{2} a_{33}+d_{2} d_{3} a_{11}\right) \\
& +\mu_{i}\left[\left(a_{11} a_{33}-a_{31} a_{13}\right) d_{2}-a_{12} a_{21} d_{3}\right]+a_{12} a_{21} a_{33} .
\end{aligned}
$$

Denote

$$
A_{1}=-\left(a_{11}+a_{33}\right), \quad A_{2}=a_{11} a_{33}-a_{12} a_{21}-a_{13} a_{31}, \quad A_{3}=a_{12} a_{21} a_{33} .
$$

Thus, $A_{1}, A_{2}, A_{3}, B_{1 i}, B_{2 i}, B_{3 i}$ are positive. Furthermore, we can calculate that

$$
B_{1 i} B_{2 i}-B_{3 i}=R_{1} \mu_{i}^{3}+R_{2} \mu_{i}^{2}+R_{3} \mu_{i}+A_{1} A_{2}-A_{3},
$$

with

$$
R_{1}=\left(d_{1}+d_{2}+d_{3}\right)\left(d_{1} d_{2}+d_{2} d_{3}+d_{1} d_{3}\right)-d_{1} d_{2} d_{3}>0,
$$




$$
\begin{aligned}
R_{2}= & -\left(a_{11}+a_{33}\right)\left(d_{1} d_{2}+d_{2} d_{3}+d_{1} d_{3}\right) \\
& -\left(d_{1}+d_{2}+d_{3}\right)\left[a_{33} d_{1}+\left(a_{11}+a_{33}\right) d_{2}+a_{11} d_{3}\right]+d_{1} d_{2} a_{33}+d_{2} d_{3} a_{11} \\
= & -\left(a_{11}+a_{33}\right)\left(2 d_{1} d_{2}+2 d_{2} d_{3}+d_{2}^{2}+d_{1} d_{3}\right)-\left(a_{33} d_{1}+a_{11} d_{3}\right)\left(d_{1}+d_{3}\right) \\
> & 0, \\
R_{3}= & \left(d_{1}+d_{2}+d_{3}\right)\left(a_{11} a_{33}-a_{12} a_{21}-a_{13} a_{31}\right) \\
& +\left(a_{11}+a_{33}\right)\left[a_{33} d_{1}+\left(a_{11}+a_{33}\right) d_{2}+a_{11} d_{3}\right] \\
& -\left[\left(a_{11} a_{33}-a_{13} a_{31}\right) d_{2}-a_{12} a_{21} d_{3}\right] \\
= & \left(2 a_{11} a_{33}-a_{12} a_{21}+a_{33}^{2}-a_{13} a_{31}\right) d_{1}+\left(2 a_{11} a_{33}-a_{12} a_{21}+a_{11}^{2}+a_{33}^{2}\right) d_{2} \\
& +\left(2 a_{11} a_{33}-a_{13} a_{31}+a_{11}^{2}\right) d_{3}>0 .
\end{aligned}
$$

Then

$$
\begin{aligned}
A_{1} A_{2}-A_{3} & =-\left(a_{11}+a_{33}\right)\left(a_{11} a_{33}-a_{12} a_{21}-a_{13} a_{31}\right)-a_{12} a_{21} a_{33} \\
& =-a_{11}^{2} a_{33}+a_{11} a_{12} a_{21}+a_{11} a_{13} a_{31}-a_{11} a_{33}^{2}+a_{13} a_{31} a_{33}>0,
\end{aligned}
$$

and $B_{1 i} B_{2 i}-B_{3 i}>0$ for all $i \geq 0$. The Routh-Hurwitz criterion shows that for each $i \geq 0$, the three roots $\lambda_{i, 1}, \lambda_{i, 2}, \lambda_{i, 3}$ of (2.4) all have negative real parts.

Moreover, it is easy to see that there exists a positive constant $\delta$ such that $\operatorname{Re}\left\{\lambda_{i, 1}\right\}, \operatorname{Re}\left\{\lambda_{i, 2}\right\}, \operatorname{Re}\left\{\lambda_{i, 3}\right\} \leq-\delta, i \geq 0$. Consequently, the spectral of $L$, consisting only of eigenvalues, lies in $\{\operatorname{Re} \lambda \leq-\delta\}$, and then the local stability of $\mathbf{u}^{*}$ follows by applying Theorem 5.1.1 of Henry [12].

Summarizing the arguments above, we have the following theorem.

Theorem 2.1. Assume that the condition (1.3) and (2.3) hold. Then the positive constant steady state $\mathbf{u}^{*}$ of $(1.4)$ is locally asymptotically stable.

Next, we consider the global stability of the positive constant steady state $\mathbf{u}^{*}$ for (1.4), which implies that the three species of prey and predator will be spatially homogeneously distributed as time converges to infinities. To this end, we assume that

$$
G(B+e-b)^{2}[b(e-b)-e]+A(e-b)^{2}(B-b)[(E-B)-(e-b)]<0 .
$$

Theorem 2.2. Suppose that the conditions (1.3) and (2.5) hold. Then the positive constant steady state $\mathbf{u}^{*}$ is globally asymptotically stable.

Proof. Let $(u(x, t), v(x, t), w(x, t))$ be a positive solution of (1.4). To prove our statement, it suffices to construct a proper Lyapunov function. For that purpose, adapting the Lyapunov function in [29], we define

$$
W(u, v, w)=\int_{u^{*}}^{u} \frac{u-u^{*}}{u} \mathrm{~d} u+\beta \int_{v^{*}}^{v} \frac{v-v^{*}}{v} \mathrm{~d} v+\gamma \int_{w^{*}}^{w} \frac{w-w^{*}}{w} \mathrm{~d} w,
$$

and

$$
E(t)=\int_{\Omega} W(u(x, t), v(x, t), w(x, t)) \mathrm{d} x
$$


where $\beta, \gamma$ are positive constants to be determined. Simple computations yield that

$$
\begin{aligned}
& \frac{\mathrm{d} E(t)}{\mathrm{d} t} \\
= & \int_{\Omega}\left\{W_{u} u_{t}+W_{v} v_{t}+W_{w} w_{t}\right\} \mathrm{d} x \\
= & \int_{\Omega}\left\{\frac{u-u^{*}}{u}\left[d_{1} \Delta u+u\left(1-u-\frac{a v}{1+b u}-\frac{A w}{1+B u}\right)\right]\right. \\
& +\beta \frac{v-v^{*}}{v}\left[d_{2} \Delta v+d v\left(-1+\frac{e u}{1+b u}\right)\right] \\
& \left.+\gamma \frac{w-w^{*}}{w}\left[d_{3} \Delta w+D w\left(-1-G w+\frac{E u}{1+B u}\right)\right]\right\} \mathrm{d} x \\
= & \int_{\Omega}\left(d_{1} \frac{u-u^{*}}{u} \Delta u+\beta d_{2} \frac{v-v^{*}}{v} \Delta v+\gamma d_{3} \frac{w-w^{*}}{w} \Delta w\right) \mathrm{d} x \\
& +\int_{\Omega}\left\{\left(u-u^{*}\right)\left(1-u-\frac{a v}{1+b u}-\frac{A w}{1+B u}\right)\right. \\
& \left.+d \beta\left(v-v^{*}\right)\left(-1+\frac{e u}{1+b u}\right)+D \gamma\left(w-w^{*}\right)\left(-1-G w+\frac{E u}{1+B u}\right)\right\} \mathrm{d} x \\
= & I_{1}(t)+I_{2}(t),
\end{aligned}
$$

in which the definitions of $I_{1}(t)$ and $I_{2}(t)$ are clear.

Due to the homogeneous Neumann boundary condition, it is evident that

$$
I_{1}(t)=-\int_{\Omega}\left\{d_{1} \frac{u^{*}}{u^{2}}|\nabla u|^{2}+d_{2} \beta \frac{v^{*}}{v^{2}}|\nabla v|^{2}+d_{3} \gamma \frac{w^{*}}{w^{2}}|\nabla w|^{2}\right\} \mathrm{d} x \leq 0 .
$$

Furthermore,

$$
\begin{aligned}
I_{2}(t)= & \int_{\Omega}\left\{\left(u-u^{*}\right)\left[-\left(u-u^{*}\right)+\frac{a v^{*}}{1+b u^{*}}-\frac{a v}{1+b u}+\frac{A w^{*}}{1+B u^{*}}-\frac{A w}{1+B u}\right]\right. \\
& +\beta d\left(v-v^{*}\right)\left(\frac{e u}{1+b u}-\frac{e u^{*}}{1+b u^{*}}\right) \\
& \left.+\gamma D\left(w-w^{*}\right)\left(G w^{*}-G w+\frac{E u}{1+B u}-\frac{E u^{*}}{1+B u^{*}}\right)\right\} \mathrm{d} x \\
= & \int_{\Omega}\left\{\left(u-u^{*}\right)^{2}\left[-1+\frac{a b v^{*}}{(1+b u)\left(1+b u^{*}\right)}+\frac{A B w^{*}}{(1+B u)\left(1+B u^{*}\right)}\right]\right. \\
& +\frac{1}{1+b u}\left[-a+\beta d e-\frac{\beta b d e u^{*}}{1+b u^{*}}\right]\left(u-u^{*}\right)\left(v-v^{*}\right) \\
& +\frac{1}{1+B u}\left[-A+\gamma D E-\frac{\gamma B D E u^{*}}{1+B u^{*}}\right]\left(u-u^{*}\right)\left(w-w^{*}\right) \\
& \left.-\gamma G D\left(w-w^{*}\right)^{2}\right\} \mathrm{d} x .
\end{aligned}
$$


Let $\beta=\frac{a}{(e-b) d}, \gamma=\frac{A(B+e-b)}{E D(e-b)}$. Then

$$
\begin{gathered}
I_{2}(t)=\int_{\Omega}\left\{\left(u-u^{*}\right)^{2}\left(-1+\frac{a b v^{*}}{(1+b u)\left(1+b u^{*}\right)}+\frac{A B w^{*}}{(1+B u)\left(1+B u^{*}\right)}\right)\right. \\
\left.-\frac{A G(B+e-b)}{E(e-b)}\left(w-w^{*}\right)^{2}\right\} \mathrm{d} x .
\end{gathered}
$$

Under the assumption (2.5), we obtain

$-1+\frac{a b v^{*}}{(1+b u)\left(1+b u^{*}\right)}+\frac{A B w^{*}}{(1+B u)\left(1+B u^{*}\right)} \leq-1+\frac{a b v^{*}}{1+b u^{*}}+\frac{A B w^{*}}{1+B u^{*}}<0$.

Hence, $I_{2}(t) \leq 0$, and thus $\frac{\mathrm{d} E(t)}{\mathrm{d} t}=I_{1}(t)+I_{2}(t) \leq 0$, which implies the desired assertion since the equality holds only when $(u, v, w)=\left(u^{*}, v^{*}, w^{*}\right)$. The proof is complete.

\section{Nonexistence of nonconstant positive steady states}

In this section, we shall discuss the nonexistence of nonconstant positive solutions of (1.5), which indicates the effect of large diffusivity. Firstly, we give a priori positive upper bounds for the positive solutions of elliptic problem (1.5). For the aim, we first cite two lemmas which are due to Lin, $\mathrm{Ni}$ and Takagi [21], and Lou and Ni [23], respectively.

Lemma 3.1 (Harnack Inequality [21]). Assume that $c \in C(\bar{\Omega})$ and let $w \in$ $C^{2}(\Omega) \cap C^{1}(\bar{\Omega})$ be a positive solution to $\Delta w(x)+c(x) w(x)=0$ in $\Omega, \frac{\partial w}{\partial \nu}=0$ on $\partial \Omega$. Then there exists a positive constant $C_{*}=C_{*}\left(\|c\|_{\infty}\right)$ such that $\max _{\bar{\Omega}} w \leq$ $C_{*} \min _{\Omega} w$.

Lemma 3.2 (Maximum Principle [23]). Suppose that $g \in C(\bar{\Omega} \times \mathbb{R})$.

(i) Assume that $w \in C^{2}(\Omega) \cap C^{1}(\bar{\Omega})$ satisfies $\Delta w(x)+g(x, w(x)) \geq 0$ in $\Omega$ and $\frac{\partial w}{\partial \nu} \leq 0$ on $\partial \Omega$. If $w\left(x_{0}\right)=\max _{\bar{\Omega}} w$, then $g\left(x_{0}, w\left(x_{0}\right)\right) \geq 0$.

(ii) Assume that $w \in C^{2}(\Omega) \cap C^{1}(\bar{\Omega})$ satisfies $\Delta w(x)+g(x, w(x)) \leq 0$ in $\Omega$ and $\frac{\partial w}{\partial \nu} \geq 0$ on $\partial \Omega$. If $w\left(x_{0}\right)=\min _{\bar{\Omega}} w$, then $g\left(x_{0}, w\left(x_{0}\right)\right) \leq 0$.

Throughout the rest of this paper, the solutions refers to the classical solution, by which we mean a solution in $C^{2}(\Omega) \cap C^{1}(\bar{\Omega})$. By virtue of the standard regularity theory for elliptic equations ([11]), Lemma 3.1 and Lemma 3.2 can be applied to system (1.5). For notational convenience, we write $\Lambda=\Lambda(a, b, d, e, A, B, D, E, G)$. In the next paragraph, the generic constants, $C_{*}, \bar{C}, C_{0}, L_{1}, L_{2}$, will depend on the domain $\Omega$ and the dimension $N$. However, as $\Omega$ and $N$ are fixed, we will not mention the dependence explicitly.

Theorem 3.3. Let $d^{*}$ be a fixed positive number and $E-B-1>0$. If $d_{i} \geq d^{*}(i=1,2,3)$ hold, then there exist positive constants $C^{*}=C^{*}\left(\Lambda, d^{*}\right)$ and $\bar{C}=\bar{C}\left(\Lambda, d^{*}\right)$ such that positive solution $(u, v, w)$ of $(1.5)$ satisfies

$$
\max _{\bar{\Omega}} u \leq \bar{C} \min _{\bar{\Omega}} u, \quad \max _{\bar{\Omega}} v \leq \bar{C} \min _{\bar{\Omega}} v, \quad \max _{\bar{\Omega}} w \leq \bar{C} \min _{\bar{\Omega}} w
$$




$$
\max _{\bar{\Omega}} u \leq 1, \quad \max _{\bar{\Omega}} v \leq C^{*}, \quad \max _{\bar{\Omega}} w \leq \frac{E-B-1}{G(1+B)} .
$$

Proof. Firstly, a direct application of the maximum principle to the first equation of (1.5) gives $u \leq 1$ on $\bar{\Omega}$, since

$$
u(1-u)-\frac{a u v}{1+b u}-\frac{A u w}{1+B u} \leq u(1-u) .
$$

It further implies that

$$
D w\left(-1-G w+\frac{E u}{1+B u}\right) \leq D w\left(-1-G w+\frac{E}{1+B}\right),
$$

which yields the third inequality of (3.2) by applying the maximum principle again.

Let $u_{0}=\max _{\bar{\Omega}} u$. From the first equation of (1.5) and the maximum principle, we obtain

$$
1-u\left(x_{0}\right)-\frac{a v\left(x_{0}\right)}{1+b u\left(x_{0}\right)}-\frac{A w\left(x_{0}\right)}{1+B u\left(x_{0}\right)} \geq 0,
$$

and thus,

$$
\frac{a v\left(x_{0}\right)}{1+b u\left(x_{0}\right)} \leq 1-u\left(x_{0}\right)-\frac{A w\left(x_{0}\right)}{1+B u\left(x_{0}\right)} \leq 1 .
$$

Hence, $v\left(x_{0}\right) \leq(1+b) / a$. Let

$$
-\Delta v=\frac{v}{d_{2}}\left(-d+\frac{e u}{1+b u}\right):=c_{2}(x) v
$$

Then, when $d_{2} \geq d^{*}$, we get

$$
\left|c_{2}(x)\right|=\left|\frac{d}{d_{2}}\left(-1+\frac{e u}{1+b u}\right)\right| \leq \frac{d}{d^{*}}\left(1+\frac{e}{1+b}\right) .
$$

It follows from the Harnack inequality that there exists a positive constant $C_{2}$ such that

$$
\max _{\bar{\Omega}} v \leq C_{2} \min _{\bar{\Omega}} v \leq C_{2} \frac{1+b}{a}:=C^{*} .
$$

In order to prove the first and second inequality of (3.2), we define

$$
\begin{aligned}
& c_{1}(x)=\frac{1}{d_{1}}\left(1-u-\frac{a v}{1+b u}-\frac{A w}{1+B u}\right), \\
& c_{3}(x)=\frac{D}{d_{3}}\left(-1-G w+\frac{E u}{1+B u}\right) .
\end{aligned}
$$

Then, the inequalities of (3.1) indicate that there exists a positive constant $C_{0}=C_{0}\left(\Lambda, d^{*}\right)$ such that $\left\|c_{1}\right\|_{\infty} \leq C_{0},\left\|c_{2}\right\|_{\infty} \leq C_{0}$, when $d_{1} \geq d^{*}$ and $d_{3} \geq d^{*}$. Hence, by applying the Harnack inequality, then there exists a positive constant $\bar{C}=\bar{C}\left(\Lambda, d^{*}\right)$ such that

$$
\max _{\bar{\Omega}} u \leq \bar{C} \min _{\bar{\Omega}} u, \quad \max _{\bar{\Omega}} w \leq \bar{C} \min _{\bar{\Omega}} w ;
$$


The proof is completed.

Theorem 3.4. Let $d_{2}^{*}$ and $d_{3}^{*}$ be fixed positive constants and satisfy

$$
d_{2}^{*} \mu_{1}>\frac{d(e-b-1)}{1+b}, \quad d_{3}^{*} \mu_{1}>\frac{D(E-B-1)}{1+B} .
$$

Then there exists a positive constant $D_{1}=D_{1}\left(\Lambda, d_{2}^{*}, d_{3}^{*}\right)$ such that (1.5) has no nonconstant positive solution provided that $d_{1}>D_{1}, d_{2} \geq d_{2}^{*}$ and $d_{3} \geq d_{3}^{*}$.

Proof. Assume that $(u, v, w)$ is a positive solution of (1.5). Let $\bar{\varphi}=\frac{1}{|\Omega|} \int_{\Omega} \varphi \mathrm{d} x$ for any $\varphi \in L^{1}(\Omega)$. Multiplying $(\mathbf{u}-\overline{\mathbf{u}})$ to the first equation in (1.5) and integrating over $\Omega$ by parts, we have that

$$
\begin{aligned}
& \int_{\Omega} d_{1}|\nabla u|^{2} \mathrm{~d} x \\
= & \int_{\Omega}\left[G_{1}(\mathbf{u})-G_{1}(\overline{\mathbf{u}})\right](u-\bar{u}) \mathrm{d} x \\
= & \int_{\Omega}\left[u(1-u)-\frac{a u v}{1+b u}-\frac{A u w}{1+B u}\right. \\
& \left.\quad-\left(\bar{u}(1-\bar{u})-\frac{a \bar{u} \bar{v}}{1+b \bar{u}}-\frac{A \bar{u} \bar{w}}{1+B \bar{u}}\right)\right](u-\bar{u}) \mathrm{d} x \\
= & \int_{\Omega}\left[(u-\bar{u})^{2}[1-(u+\bar{u})]-\frac{(a b u \bar{u}+a \bar{u})(u-\bar{u})(v-\bar{v})+a v(u-\bar{u})^{2}}{(1+b u)(1+b \bar{u})}\right. \\
& \left.\quad-\frac{(A B u \bar{u}+A \bar{u})(u-\bar{u})(w-\bar{w})+A w(u-\bar{u})^{2}}{(1+B u)(1+B \bar{u})}\right] \mathrm{d} x .
\end{aligned}
$$

In a similar way, we can obtain that

$$
\begin{aligned}
\int_{\Omega} d_{2}|\nabla v|^{2} \mathrm{~d} x & =\int_{\Omega}\left[G_{2}(\mathbf{u})-G_{2}(\overline{\mathbf{u}})\right](v-\bar{v}) \mathrm{d} x \\
& =\int_{\Omega}\left[d v\left(-1+\frac{e u}{1+b u}\right)-d \bar{v}\left(-1+\frac{e \bar{u}}{1+b \bar{u}}\right)\right](v-\bar{v}) \mathrm{d} x \\
& =\int_{\Omega}\left[-d(v-\bar{v})^{2}+\frac{d e \bar{u}(1+b u)(v-\bar{v})^{2}}{(1+b u)(1+b \bar{u})}+\frac{d e v(u-\bar{u})(v-\bar{v})}{(1+b u)(1+b \bar{u})}\right] \mathrm{d} x
\end{aligned}
$$

and

$$
\begin{aligned}
\int_{\Omega} d_{3}|\nabla w|^{2} \mathrm{~d} x= & \int_{\Omega}\left[G_{3}(\mathbf{u})-G_{3}(\overline{\mathbf{u}})\right](w-\bar{w}) \mathrm{d} x \\
= & \int_{\Omega}\left[D w\left(-1-G w+\frac{E u}{1+B u}\right)\right. \\
& \left.\quad-D \bar{w}\left(-1-G \bar{w}+\frac{E \bar{u}}{1+B \bar{u}}\right)\right](w-\bar{w}) \mathrm{d} x \\
= & \int_{\Omega}\left[-[D+G(w+\bar{w})](w-\bar{w})^{2}+\frac{D E \bar{u}(1+B u)(w-\bar{w})^{2}}{(1+B u)(1+B \bar{u})}\right.
\end{aligned}
$$




$$
\left.+\frac{D E w(u-\bar{u})(w-\bar{w})}{(1+B u)(1+B \bar{u})}\right] \mathrm{d} x .
$$

What we have done implies the following estimates:

$$
\begin{aligned}
& \int_{\Omega}\left\{d_{1}|\nabla u|^{2}+d_{2}|\nabla v|^{2}+d_{3}|\nabla w|^{2}\right\} \mathrm{d} x \\
\leq & \int_{\Omega}\left[(u-\bar{u})^{2}-d(v-\bar{v})^{2}+\frac{d e \bar{u}(1+b u)(v-\bar{v})^{2}}{(1+b u)(1+b \bar{u})}+\frac{d e v(u-\bar{u})(v-\bar{v})}{(1+b u)(1+b \bar{u})}\right. \\
& \left.-D(w-\bar{w})^{2}+\frac{D E \bar{u}(1+B u)(w-\bar{w})^{2}}{(1+B u)(1+B \bar{u})}+\frac{D E w(u-\bar{u})(w-\bar{w})}{(1+B u)(1+B \bar{u})}\right] \mathrm{d} x \\
\leq & \int_{\Omega}\left[(u-\bar{u})^{2}-d(v-\bar{v})^{2}+\frac{d e}{1+b}(v-\bar{v})^{2}+\frac{d e v(u-\bar{u})(v-\bar{v})}{(1+b u)(1+b \bar{u})}\right. \\
& \left.-D(w-\bar{w})^{2}+\frac{D E}{1+B}(w-\bar{w})^{2}+\frac{D E w(u-\bar{u})(w-\bar{w})}{(1+B u)(1+B \bar{u})}\right] \mathrm{d} x \\
\leq & \int_{\Omega}\left[(u-\bar{u})^{2}-d(v-\bar{v})^{2}+\frac{d e}{1+b}(v-\bar{v})^{2}+2 L_{1}|u-\bar{u}||v-\bar{v}|\right. \\
& \left.-D(w-\bar{w})^{2}+\frac{D E}{1+B}(w-\bar{w})^{2}+2 L_{2}|u-\bar{u}||w-\bar{w}|\right] \mathrm{d} x \\
\leq & \int_{\Omega}\left[(u-\bar{u})^{2}+\frac{d(e-b-1)}{1+b}(v-\bar{v})^{2}+2 L_{1}|u-\bar{u} \| v-\bar{v}|\right. \\
& \left.+\frac{D(E-B-1)}{1+B}(w-\bar{w})^{2}+2 L_{2}|u-\bar{u}||w-\bar{w}|\right] \mathrm{d} x \\
\leq & \int_{\Omega}\left[(u-\bar{u})^{2}\left(1+\frac{L_{1}}{\epsilon_{1}}+\frac{L_{2}}{\epsilon_{2}}\right)+(v-\bar{v})^{2}\left(\frac{d(e-b-1)}{1+b}+\epsilon_{1} L_{1}\right)\right. \\
& \left.+(w-\bar{w})^{2}\left(\frac{D(E-B-1)}{1+B}+\epsilon_{2} L_{2}\right)\right] \mathrm{d} x
\end{aligned}
$$

for some positive constants $L_{1}, L_{2}$ and arbitrarily small positive constants $\epsilon_{1}$, $\epsilon_{2}$, in which the last inequality was obtained by the following fact,

$$
\begin{aligned}
& 2 L_{1}|u-\bar{u}||v-\bar{v}|=2 \sqrt{\frac{L_{1}}{\epsilon_{1}}}|u-\bar{u}| \cdot \sqrt{\epsilon_{1} L_{1}}|v-\bar{v}| \leq \frac{L_{1}}{\epsilon_{1}}|u-\bar{u}|^{2}+\epsilon_{1} L_{1}|v-\bar{v}|^{2}, \\
& 2 L_{2}|u-\bar{u}||w-\bar{w}|=2 \sqrt{\frac{L_{2}}{\epsilon_{2}}}|u-\bar{u}| \cdot \sqrt{\epsilon_{2} L_{2}}|w-\bar{w}| \leq \frac{L_{2}}{\epsilon_{2}}|u-\bar{u}|^{2}+\epsilon_{2} L_{2}|w-\bar{w}|^{2} .
\end{aligned}
$$

Applying the Poincáre inequality, it is clear that

$$
\begin{aligned}
& \int_{\Omega}\left\{d_{1} \mu_{1}|u-\bar{u}|^{2}+d_{2} \mu_{1}|v-\bar{v}|^{2}+d_{3} \mu_{1}|w-\bar{w}|^{2}\right\} \mathrm{d} x \\
\leq & \int_{\Omega}\left\{(u-\bar{u})^{2}\left(1+\frac{L_{1}}{\epsilon_{1}}+\frac{L_{2}}{\epsilon_{2}}\right)+(v-\bar{v})^{2}\left(\frac{d(e-b-1)}{1+b}+\epsilon_{1} L_{1}\right)\right. \\
& \left.+(w-\bar{w})^{2}\left(\frac{D(E-B-1)}{1+B}+\epsilon_{2} L_{2}\right)\right\} \mathrm{d} x .
\end{aligned}
$$


From the assumption, we can find sufficiently small constants $\epsilon_{1,0}, \epsilon_{2,0}>0$ such that

$$
d_{2}^{*} \mu_{1} \geq \frac{d(e-b-1)}{1+b}+\epsilon_{1,0} L_{1}, \quad d_{3}^{*} \mu_{1} \geq \frac{D(E-B-1)}{1+B}+\epsilon_{2,0} L_{2} .
$$

Finally, by taking $D_{1}:=\frac{1}{\mu_{1}}\left(1+\frac{L_{1}}{\epsilon_{1,0}}+\frac{L_{2}}{\epsilon_{2,0}}\right)$, one can conclude that when $d_{1}>D_{1}$, then $u=\bar{u}, v=\bar{v}$ and $w=\bar{w}$ hold. This completes the proof.

\section{Concluding remarks}

In this paper, we study a diffusive two predators and one prey model with Holling type II functional response under homogeneous Neumann boundary conditions. From Theorem 2.2, we can see that under suitable conditions of the parameters, the unique positive constant equilibrium $\mathbf{u}^{*}$ of system (1.4) is globally asymptotically stable which is coincident with the results of the corresponding model without diffusion studied in [29]. In this case, the free diffusion does not affect the global stability of the unique constant positive equilibrium and thus nonconstant positive solutions do no exist. However, when the diffusion coefficients are large enough, i.e., the case of large diffusivity, we can see that the diffusion has a certain influence on the population evolution. From Theorem 3.4, under proper conditions concerned with the diffusion coefficients $d_{2}$ and $d_{3}$, there is no nonconstant positive steady state provided that diffusion coefficient $d_{1}$ of the prey is sufficiently large. These results seem to be coincident with the natural phenomena in ecological systems. When the prey species diffuses fast, the coexistence state is exactly the constant positive steady state. In this situation, the system is similar to the spatially homogeneous case. On the contrary, if the predator species diffuses fast, then at some spatial locations, the coexistence state may be different from the constant positive steady state, that is to say, how to give a priori positive lower bounds for the positive solutions of elliptic problem (1.5) and establish the existence of nonconstant positive steady states is worth further investigations.

Acknowledgement. The author would like to thank the anonymous referees and the editors for their suggestions which greatly improved the presentation of this paper.

\section{References}

[1] D. G. Aronson and H. F. Weinberger, Nonlinear diffusion in population genetics, combustion, and nerve pulse propagation, In Partial Differential Equations and Related Topics, (Edited by J. A. Goldstein), Lecture Notes in Mathematics, Vol. 446, pp. 5-49, Springer, Berlin, 1975.

[2] A. D. Bazykin, Nonlinear Dynamics of Interacting Populations, World Scientific, Singapore, 1998.

[3] J. R. Beddington, Mutual interference between parasites or predators and its effect on searching efficiency, J. Animal Ecol. 44 (1975), no. 1, 331-340.

[4] R. S. Cantrell and G. C. Cosner, Spatial Ecology via Reaction-Diffusion Equations, John Wiley \& Sons, Chichester, UK, 2003. 
[5] D. L. DeAngelis, R. A. Goldstein, and R. V. O'Neill, A model for trophic interaction, Ecology 56 (1975), no. 4, 881-892.

[6] O. Diekmann, Thresholds and travelling waves for the geographical spread of infection, J. Math. Biol. 6 (1978), no. 2, 109-130.

[7] Y. Du and Y. Lou, Some uniqueness and exact multiplicity results for a predator-prey model, Trans. Amer. Math. Soc. 349 (1997), no. 6, 2443-2475.

[8] - Qualitative behavior of positive solutions of a predator-prey model: effects of saturation, Proc. Roy. Soc. Edinb. 131A (2001), no. 2, 321-349.

[9] Y. Du and J. Shi, Some recent results on diffusive predator-prey models in spatially heterogeneous envirment, In Nonlinear dynamics and evolution equations (Ed. by $\mathrm{H}$. Brunner, X. Zhao and X. Zou), 95-135, Fields Inst. Commun. 48, AMS, Providence, RI, 2006.

[10] Y. H. Fan and W. T. Li, Global asymptotic stability of a ratio-dependent predator-prey system with diffusion, J. Comput. Appl. Math. 188 (2006), no. 2, 205-227.

[11] D. Gilbarg and N. S. Trudinger, Elliptic Partial Differential Equations of Second Order, Springer-Verlag, 2001.

[12] D. Henry, Geometric Theory of Semilinear Parabolic Equations, Lecture Notes in Mathematics, Vol. 840, Springer-Verlag, Berlin, New York, 1981.

[13] M. A. Hixon and G. P. Jones, Competition, predation, and density-dependent mortality in demersal marine fishes, Ecology 86 (2005), no. 11, 2847-2859.

[14] C. S. Holling, The functional response of predator to prey density and its role in mimicry and population regulation, Mem. Entomol. Soc. Can. 97 (1965), no. S45, 5-60.

[15] W. Ko and I. Ahn, Analysis of ratio-dependent food chain model, J. Math. Anal. Appl. 335 (2007), no. 1, 498-523.

[16] W. Ko and K. Ryu, Qualitative analysis of a predator-prey model with Holling type II functional response incorporating a prey refuge, J. Differential Equations 231 (2006), no. 2, 534-550.

[17] - A qualitative study on general Gause-type predator-prey models with constant diffusion rates, J. Math. Anal. Appl. 344 (2008), no. 1, 217-230.

[18] — A qualitative study on general Gause-type predator-prey models with nonmonotonic functional response, Nonlinear Anal. Real World Appl. 10 (2009), no. 4, 2558-2573.

[19] L. Li, Coexistence theorems of steady states for predator-prey interacting systems, Trans. Amer. Math. Soc. 305 (1988), no. 1, 143-166.

[20] Z. Lin and M. Pederson, Stability in a diffusive food-chain model with Michaelis-Menten functional response, Nonlinear Anal. 57 (2004), no. 3, 421-433.

[21] L. Lin, W. M. Ni, and I. Takagi, Large amplitude stationary solutions to a chemotaxis systems, J. Differential Equations 72 (1988), no. 1, 1-27.

[22] A. J. Lotka, Elements of Physical Biology, Williams and Wilkins Company, Baltimore, 1925.

[23] Y. Lou and W. M. Ni, Diffusion, self-diffusion and cross-diffusion, J. Differential Equations 131 (1996), no. 1, 79-131.

[24] P. Y. H. Pang and M. Wang, Qualitative analysis of a ratio-dependent predator-prey system with diffusion, Proc. Roy. Soc. Edinburgh Sect. A 133 (2003), no. 4, 919-942.

[25] _ Strategy and stationary pattern in a three-species predator-prey, J. Differential Equations 200 (2004), no. 2, 245-273.

[26] _ Non-constant positive steady states of a predator-prey system with nonmonotonic functional response and diffusion, Proc. London Math. Soc. 88 (2004), no. $1,135-157$.

[27] P. Y. H. Pang and W. Zhou, Positive stationary solutions for a diffusive variableterritory prey-predator model, J. Math. Anal. Appl. 379 (2011), no. 1, 290-304. 
[28] R. Peng, J. Shi, and M. Wang, Stationary pattern of a ratio-dependent food chain model with diffusion, SIAM J. Appl. Math. 67 (2007), no. 5, 1479-1503.

[29] S. Ruan, A. A. P. Ricciardi, and D. L. DeAngelis, Coexistence in competition models with density-dependent mortality, C. R. Biologies 330 (2007), no. 12, 845-854.

[30] A. M. Turing, The chemical basis of morphogenesis, Phil. Trans. R. Soc. London Ser. B 237 (1952), no. 641, 37-72.

[31] V. Volterra, Fluctuations in the abundance of a species considered mathematically, Nature 118 (1926), no. 2972, 558-560.

[32] M. Wang, Stationary patterns for a prey-predator model with prey-dependent and ratiodependent functional response and diffusion, Phys. D 196 (2004), no. 1-2, 172-192.

[33] M. Wang and P. Y. H. Pang, Global asymptotic stability of positive steady states of a diffusive ratio-dependent prey-predator model, Appl. Math. Lett. 21 (2008), no. 11, 1215-1220.

School of Mathematical Science

Huaiyin Normal University

Huaian, Jiangsu 223300, P. R. China

E-mail address: hbshi2006@163.com 